\title{
Near-field diffraction-based focal length determination technique
}

\author{
Francisco Jose Torcal-Milla*, Luis Miguel Sanchez-Brea \\ Universidad Complutense de Madrid, Applied Optics Complutense Group, Optics Department, Facultad de Ciencias Fisicas, Plaza de las Ciencias, 1, 28040 \\ Madrid, Spain
}

\section{A R T I C L E I N F O}

\section{Keywords:}

Focal length

Metrology

Self-imaging

\begin{abstract}
A B S T R A C T
An accurate and simple technique for determining the focal length of a lens is presented. It consists of measuring the period of the fringes produced by a diffraction grating at the near field when it is illuminated with a beam focused by the unknown lens. In paraxial approximation, the period of the fringes varies linearly with the distance. After some calculations, a simple extrapolation of data is performed to obtain the locations of the principal plane and the focal plane of the lens. Thus, the focal length is obtained as the distance between the two mentioned planes. The accuracy of the method is limited by the collimation degree of the incident beam and by the algorithm used to obtain the period of the fringes. We have checked the technique with two commercial lenses, one convergent and one divergent, with nominal focal lengths $(+100 \pm 1) \mathrm{mm}$ and $(-100 \pm 1) \mathrm{mm}$ respectively. We have experimentally obtained the focal lengths resulting into the interval given by the manufacturer but with an uncertainty of $0.1 \%$, one order of magnitude lesser than the uncertainty given by the manufacturer.
\end{abstract}

\section{Introduction}

Accurate characterization of optical systems is crucial for applications and techniques. Among all characteristics of optical systems, the focal length is one of the most important. There are many different techniques for determining the focal length of a lens [1-4]. The simplest one consists of using a very well-collimated laser beam and determining the focal spot position by means of a screen or a twodimensional camera. Although, this technique is actually an approximation since the focal length is commonly defined from the front principal plane of the lens, and it is usually inaccessible. Other methods based on moiré deflectometry utilize two diffraction gratings and analyze the moire fringes produced after the second grating when the beam is converging through the lens [5-10]. In Refs. [11,12] two methods with a single diffraction grating are presented. In [11], the method consists of measuring the frequencies at the focal plane of the lens and obtaining the focal length for comparison with the frequencies of the grating. On the other hand, in [12] the method consists of measuring the transverse distances of diffraction maxima in one measurement at the focal plane. In Ref. [13] a Hartmann-Shack wavefront sensor is used to determine the focal plane without knowing the position of the principal plane of the lens. In Ref. [14] one diffraction grating is used to determine the focal length of a lens by measuring the demagnification of the self-images produced by the grating illuminated by a convergent beam. However, only two positions are used and then the accuracy is not optimal. Our impression is that the accuracy of the method can be greatly improved. On the other hand, Tebaldi et al. obtain the focal distance but not the location of the principal plane nor the focal plane.

As a consequence, we propose in this work an improvement of the method proposed in [14] where a very simple and accurate technique for determining the focal length of a lens or lens system is presented. It is based on the self-imaging phenomenon and consists of measuring the period of the converging/diverging self-images produced after the lens when it is illuminated by a collimated beam. On the contrary to ref. [14], our method places the grating before the lens with unknown optical parameters. Thus, it is possible to obtain the position of the principle plane of the lens. It corresponds with the plane in which the period of the self-images equals the period of the grating. Also, at the focal plane the period of the self-images is zero. Then, by extrapolating the values of the period of the self-images at several distances, it is possible to obtain the focal distance. To increase the accuracy of the technique, we use the variogram function for computing the period of the self-images since noise is highly reduced. To check the technique, we measure two lenses, one convergent and one divergent, with nominal focal lengths $+100 \mathrm{~mm}$ and $-100 \mathrm{~mm}$ respectively. The uncertainty given by the manufacturer for both lenses is $1 \%$ and we obtain an uncertainty of approximately $0.1 \%$, one order of magnitude lesser.

\footnotetext{
* Corresponding author

E-mail address: fjtorcal@ucm.es (F.J. Torcal-Milla).
} 


\section{Analytical approach}

Let us consider the set-up shown in Fig. 1. It consists of a wellcollimated light beam of wavelength $\lambda$, described by $U(z)=U_{0} e^{i k z}$ that impinges an amplitude Ronchi diffraction grating, $D G$, with wellknown period $p$. The transmittance of the grating can be expressed as its Fourier expansion series as

$t(x)=\sum_{n} a_{n} e^{i q n x_{0}}$

where $x_{0}$ is the transversal coordinate at the grating plane, $n$ are integer numbers, $a_{n}=\tau \sin c(n \pi \tau)$ are the Fourier coefficients of the grating [15], $\tau$ is the fill factor of the grating, and $q=2 \pi / p$. When the grating is illuminated by a plane wave, it produces self-images at the near field that consist of exact replicas of the intensity distribution of the grating $[16,17]$, that appear at multiples of the so-called Talbot distance, $z_{T}=2 p^{2} / \lambda$. The propagated field after the grating can be calculated by using the Fresnel approach resulting in

$U_{1}(\xi, z)=U_{0} \sqrt{\frac{i}{\lambda z}} e^{i k z} \sum_{n=-\infty}^{\infty} a_{n} e^{2 \pi i n \frac{\xi}{p}} e^{i 2 \pi n^{2} z / z T}$,

where $z$ is the distance from the grating to the observation plane. After, light propagates to the lens with unknown focal length $f^{\prime}$. Considering thin lens approximation, the transmittance of the lens centered at the optical axis in Fresnel regime is given by

$L(\xi)=e^{-i k \frac{\xi^{2}}{2 f^{\prime}}}$,

where $k=2 \pi / \lambda$. We use again Fresnel approach to propagate the optical field from the lens forward resulting in

$U_{2}(x, z)=\sqrt{\frac{i}{\lambda z}} e^{i k z} \int_{-\infty}^{+\infty} U_{1}(\xi, z) L(\xi) e^{i \frac{k}{2 z}(x-\xi)^{2}} d \xi$,

where $\xi$ is the transversal coordinate at the lens principal plane, $z$ is the distance from the front principal plane of the lens to the observation plane, and $x$ is the transversal coordinate at the observation plane. This integral is easily solved considering $\int_{-\infty}^{+\infty} \exp \left[-\left(a x^{2}+b x+c\right)\right] d x=\sqrt{\frac{\pi}{a}} \exp \left(b^{2} / 4 a-c\right)$, resulting in

$U_{2}(x, z)=U_{0} \sum_{n} a_{n} \sqrt{\frac{2 \pi}{i k\left(1 / f^{\prime}-1 / z\right)}} e^{-i \frac{k x^{2}}{2\left(f^{\prime}-z\right)} e^{-i \frac{n q f^{\prime}}{2 k\left(f^{\prime}-z\right)}(n q z-2 k x)} .}$

The intensity distribution is easily obtained $I(x, z)=U_{2}(x, z) U_{2}^{*}(x, z)$, where * denotes complex conjugated,

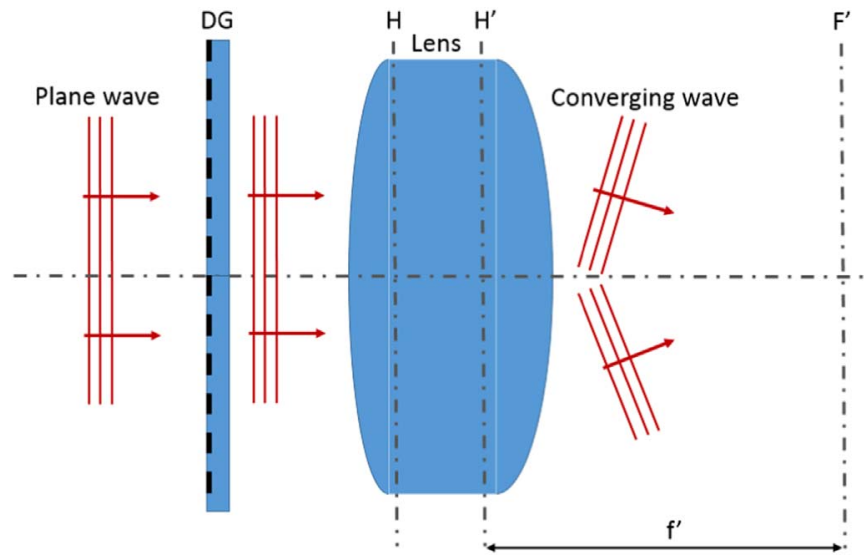

Fig. 1. Scheme of the proposed set-up for a convergent lens - similar for a divergent lens. $D G$ is the diffraction grating, $H, H^{\prime}$ are the principal planes of the lens, $F^{\prime}$ is the front focal plane, and $f^{\prime}$ is the front focal length.

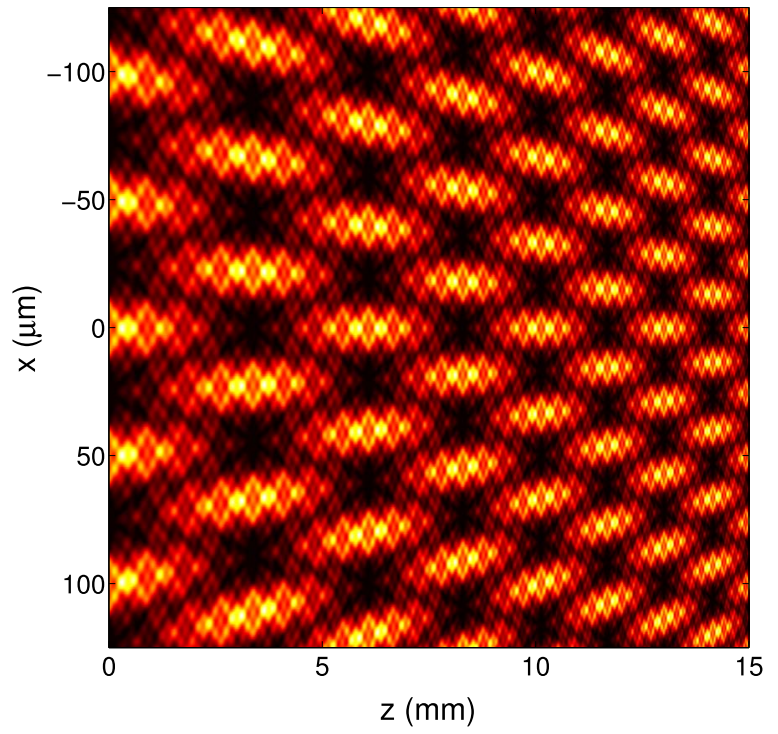

Fig. 2. Analytical near field intensity obtained with Eq. (6) by illuminating a Ronchi diffraction grating of period $p=50 \mu \mathrm{m}$ and a convergent lens of focal length $f^{\prime}=30 \mathrm{~mm}$ with a collimated beam of wavelength $\lambda=655 \mathrm{~nm}$.

$I(x, z)=I_{0} \sum_{n} \sum_{n^{\prime}} a_{n} a_{n^{\prime}}^{*} e^{i \frac{q\left(n-n^{\prime}\right) x}{1-z l f^{\prime}}} e^{-i \frac{q^{2}\left(n^{2}-n^{\prime}\right)}{2 k\left(1-z / f^{\prime}\right)},}$,

where $I_{0}$ includes all constants that do not depend on the distance $z$. The first exponential factor is related to the period of the self-images, which depends on the distance, $z$, from the principal plane as

$p^{\prime}=p\left(1-\frac{z}{f^{\prime}}\right)$

This dependence is linear and allows us to identify two points that correspond to the front focal plane, $p^{\prime}=0$, and the front principal plane of the lens, $p^{\prime}=p$.

We show in Fig. 2 an example of intensity given by Eq. (6) with a collimated beam of wavelength $\lambda=655 \mathrm{~mm}$, an amplitude diffraction Ronchi grating of period $p=50 \mu \mathrm{m}$ and a lens of focal length $f^{\prime}=30 \mathrm{~mm}$. As can be observed, Talbot self-images converge to the focal plane. It is important to notice that the period of the self-images must be calculated under paraxial approximation since the lens also curves the self-images far from the optical axis in a similar way to that is shown in $[18,19]$. Theoretically, the period has a linear dependence with $z, p^{\prime}=a z+b$. Then, after measuring the period of the fringes at each plane and calculating its linear dependence with $z$, the focal length, defined as the distance between the front principal plane and the focal plane, is obtained as

$f^{\prime}=-\frac{p}{a}$.

The uncertainty in the focal length determination is given by $\Delta f^{\prime 2}=\left(1 / a^{2}\right) \Delta p^{2}+\left(p^{2} / a^{4}\right) \Delta a^{2}$. The period of commercial gratings is given with manufacture errors around $3 \mathrm{~nm} / \mathrm{m}$. Since we are measuring in a range of a few millimeters of the grating, we can consider the period of the grating well known and therefore $\Delta p$ results negligible. Then, the uncertainty of the focal length is given by the uncertainty in the slope of the fitting of the measured periods [20]. At the end, the error in the focal length comes from errors committed in measuring the period of the fringes, $p^{\prime}$, and the corresponding distance $z$. The period of the fringes can be affected by misalignments of the optical system. Nevertheless, it can be calibrated previously since the period of the grating is well known. We can correct misalignments, simply measuring the period of the self-images without the lens. When the period of 
the self-image is that of the grating then the grating is properly aligned.

About distance $z$, it is measured with a motorized stage. In our case, we use a linear stage model M-521 by PI, which has an unidirectional repeatability of $0.1 \mu \mathrm{m}$ and a pitch of $\pm 35 \mu \mathrm{rad}$. Repeatability has been proven in several previous papers by the authors and pitch would affect the value of the measured period as a cosine error. Calculating the effect, the period would be affected by a factor $6.11 \times 10^{-10} p^{\prime}$, which results also negligible. After all these considerations, we may conclude that systematic errors can be canceled or neglected. Then, the slope of the linear regression is affected only by random errors, which we will consider in the experimental calculations in the next section.

\section{Experimental results}

The experimental set-up is similar to that shown in Fig. 1. It consists of a collimated pigtailed laser module by Monocrom, C-Series model 6707, with wavelength $\lambda=670 \mathrm{~nm}$, a Ronchi amplitude diffraction grating with a period of $p=105 \mu \mathrm{m}$, a lens and a CMOS camera fixed to a linear motorized stage. As lens under test we have used a

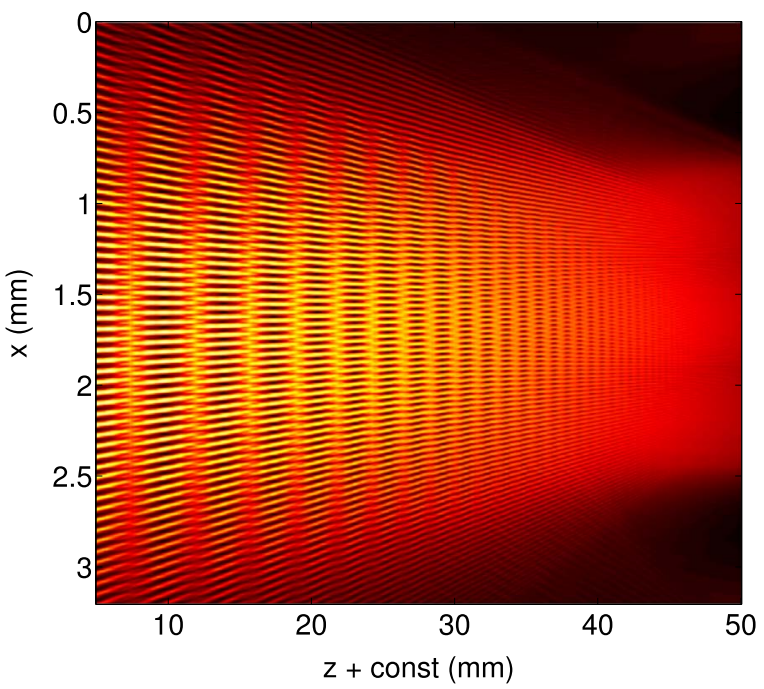

Fig. 3. Experimental intensity distribution after the converging lens KPX223 by Newport using a diffraction grating of period $p=105 \mu \mathrm{m}$ illuminated by a plane wave of wavelength $\lambda=670 \mathrm{~nm}$.
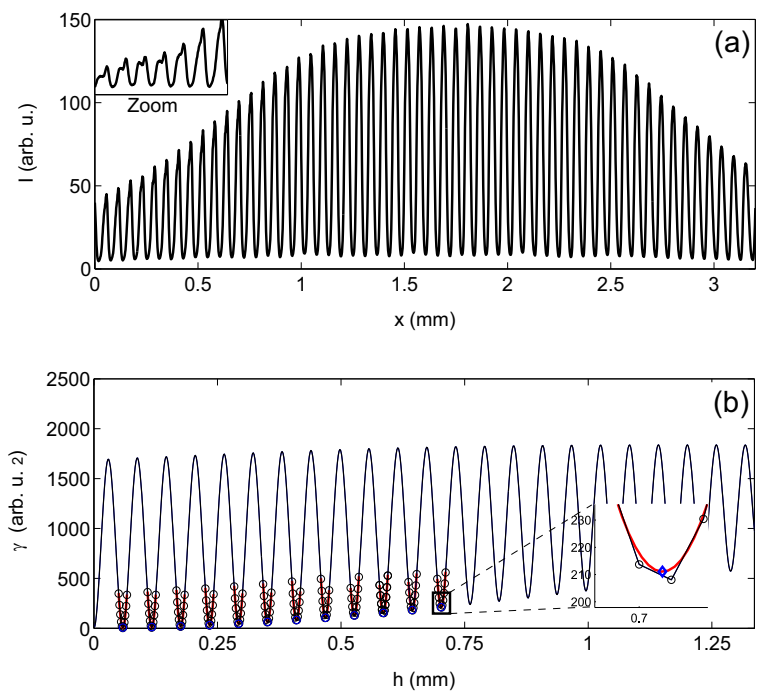

Fig. 4. (a) Profile of the first observable self-image of Fig. 3 and (b) variogram corresponding to the self-image and fitting of the minima of the variogram. Diamonds represent the minima of the fittings. (For interpretation of the references to color in this figure caption, the reader is referred to the web version of this paper.) converging lens by Newport (KPX223) with nominal focal length $f_{n}^{\prime}=100 \mathrm{~mm}$ and a divergent lens by Newport (KPC076) with nominal focal length $f_{n}^{\prime}=-100 \mathrm{~mm}$. Both lenses have an error $\Delta f_{n}^{\prime}= \pm 1 \mathrm{~mm}$ provided by the manufacturer. The experiment is similar to that shown in [21] and consists of displacing the camera along the optical axis and taking profiles of the grating diffraction fringes. We have used a CMOS camera model UI-1490LE by IDS with pixel size $1.67 \times 1.67 \mu \mathrm{m}$. We show in Fig. 3 the experimental intensity distribution obtained by displacing the camera back from the converging lens KPX223 by Newport. The initial position is unknown and that is the meaning of the constant. Each column of the figure is obtained by integrating every image parallel to the fringes. As can be observed, the self-images converge to the plane that corresponds to the focal plane, as it was expected. After measuring the fringes, we calculate the period of each experimental plane and represent them in terms of the distance $z$. We may calculate the period around even and odd self-images to increase the number of experimental points, neglecting the points at distances between Talbot planes that have half the period of the closest selfimage.

To improve the accuracy of the method, we use the variogram function to calculate the period of the fringes. It is defined as [22-24]

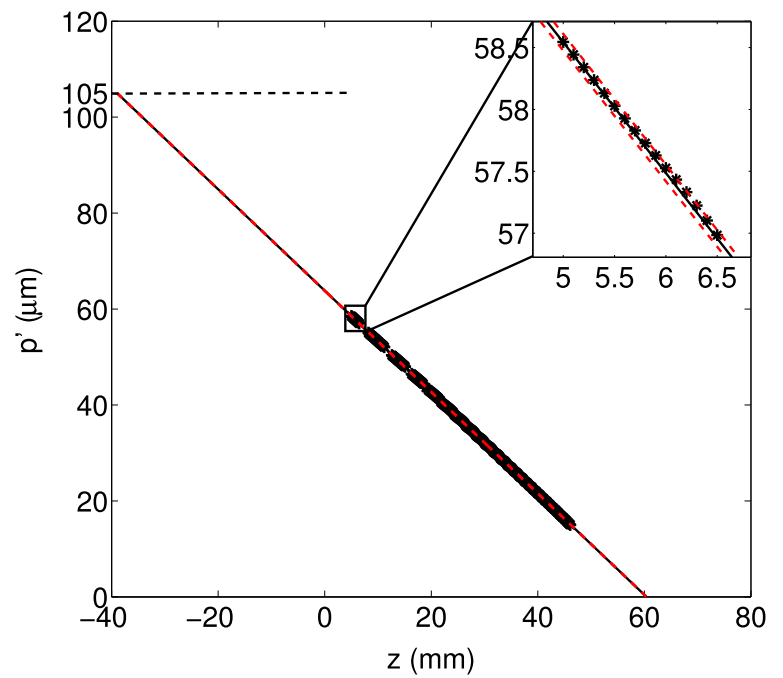

Fig. 5. Measured periods (asterisks), linear fitting (solid line) and interval of confidence (dashed line) obtained from Fig. 3. Lens model KPX223 by Newport.

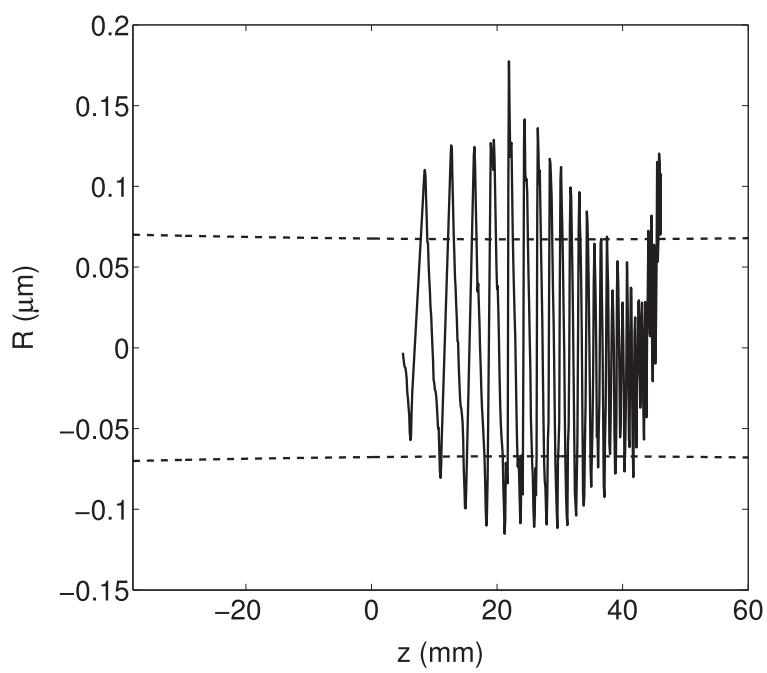

Fig. 6. Confidence interval (dashed line) and residuals $(R)$ of the fitting (solid line) shown in Fig. 5. Lens model KPX223 by Newport. 


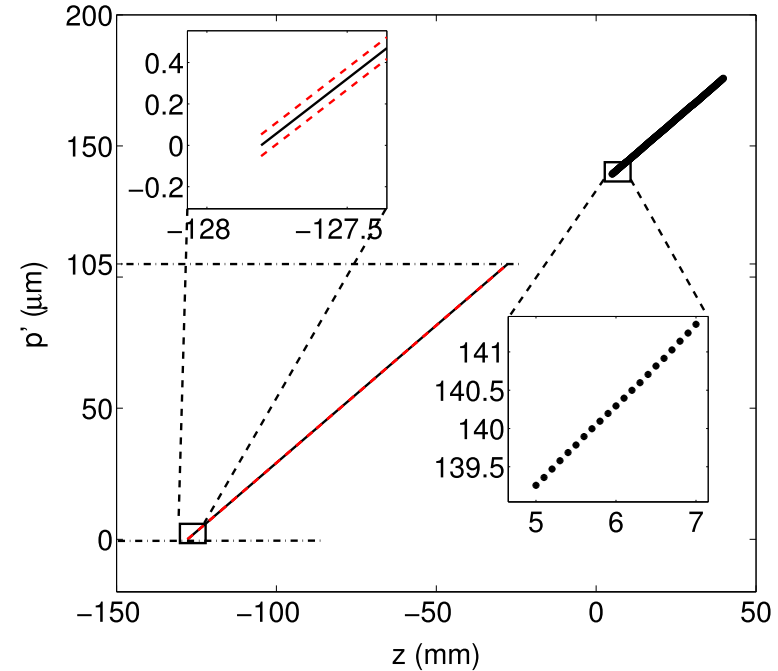

Fig. 7. Measured periods (asterisks), linear fitting (solid line) and confidence interval (dashed line). Lens KPC076 by Newport.

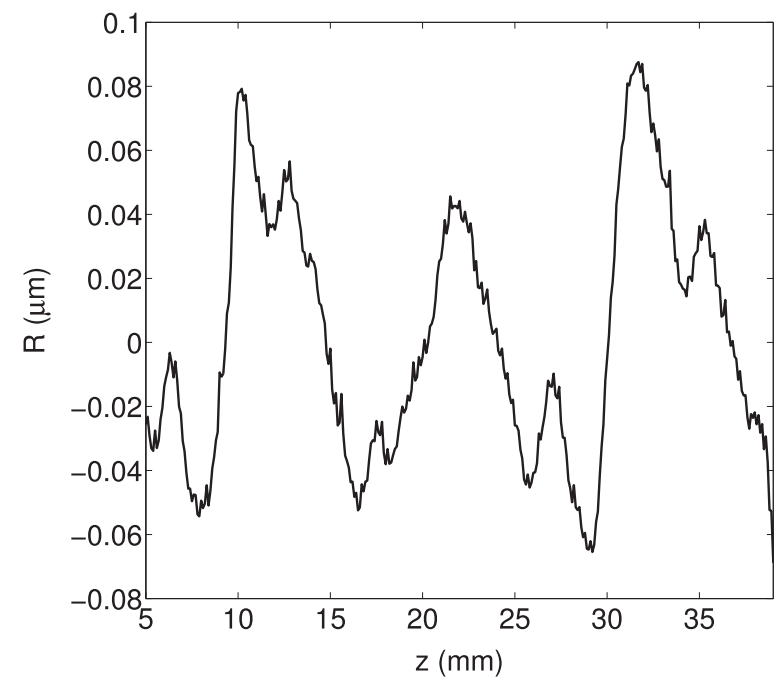

Fig. 8. Residuals $(R)$ of the fitting shown in Fig. 7. Lens KPC076 by Newport.

$2 \gamma(h)=\left\langle[I(y+h)-I(y)]^{2}\right\rangle_{y}$,

where $h$ is the distance between two data and $\langle\bullet\rangle_{y}$ means averaging with respect to $y$. When data are regularly disposed, as in a CMOS array, the variogram at $h$ is computed as

$2 \gamma(h)=\frac{1}{N(h)} \sum_{N(h)}\{I[(i+j) \Delta y]-I(i \Delta y)\}^{2}$,

where $\Delta y$ is the distance between two adjacent pixels, $i$ and $j$ are integers, and $N(h)$ is the number of pairs whose vector distance is $h=j \Delta y$. Since each data is obtained by an averaging, the variogram is much smoother than the original signal but having the same period. We take advantage of this property to calculate the periods with more accuracy.

As an example, Fig. 4 shows the profile and variogram corresponding to the first observable self-image of Fig. 3. As it was expected, the variogram function is quite smoother than the original profile but with the same period. We calculate the minima of the variogram by fitting everyone to a parabolic curve (solid red lines in Fig. 4b). In Fig. 4b we also show a zoom of one of the minima. The diamond represents the minimum obtained from the fitting. After obtaining all positions of minima of the profile, we calculate the period by averaging over them. Once we have obtained all periods corresponding to the profiles, we fit again all of them to a linear regression, taking out all points clearly out of the linear trend before fitting. This fitting is shown in Fig. 5, where asterisks are experimental points, solid line is the linear fitting and dashed lines represent the interval of confidence. Finding the distances in which the fitting is $p^{\prime}=105 \mathrm{~mm}$ and $p^{\prime}=0 \mathrm{~mm}$, respectively, and using Eq. (8), the effective focal length results $f^{\prime}=99.377 \mathrm{~mm}$ with a fitting error of $\Delta f^{\prime}= \pm 0.092 \mathrm{~mm}$. Performing the same measurements and calculations on the other side of the lens, the effective focal length results $f^{\prime}=99.549 \mathrm{~mm}$ with a fitting error of $\Delta f^{\prime}= \pm 0.072 \mathrm{~mm}$. As can be observed, both measurements are quite close and present errors in the same order of magnitude. The nominal value given by the manufacturer is $f_{n}^{\prime}=100 \mathrm{~mm}$ with error of $\Delta f_{n}^{\prime}= \pm 1 \mathrm{~mm}$. Our method improves in one order of magnitude the error given by the manufacturer and gives a more precise measurement of the effective focal length of the lens. In addition, we show in Fig. 6 the residuals corresponding to the linear fitting shown in Fig. 5. The standard deviation of the residuals results $\sigma=0.067 \mathrm{~mm}$.

To check the method for divergent lenses, we have performed the same experiment with the lens KPC076 by Newport whose nominal focal length is $f_{n}^{\prime}=-100 \mathrm{~mm}$ with error $\Delta f_{n}^{\prime}= \pm 1 \mathrm{~mm}$. We show in Fig. 7 the experimental measured periods (asterisks) and the linear fitting (solid line) with the intervals of confidence (dashed lines). In this case, the measured self-images are far from the period of the grating and the fitting must be extrapolated through the lens back to obtain the effective focal length. It results $f^{\prime}=-100.16 \mathrm{~mm}$ with a fitting error of $\Delta f^{\prime}= \pm 0.064 \mathrm{~mm}$. Performing the experiment by the other side of the lens, the effective focal length results $f^{\prime}=-100.294 \mathrm{~mm}$ with a fitting error of $\Delta f^{\prime}= \pm 0.065 \mathrm{~mm}$. Both results appear very close and with an uncertainty of around $0.1 \%$, as it resulted with the other lens under testing. The residuals of the fitting in this case are shown in Fig. 8. The standard deviation of the residuals results $\sigma=0.041 \mathrm{~mm}$. We show in Table 1 a summary with all experimental data where $f_{n}^{\prime}$ is the focal length given by the manufacturer, $H^{\prime}$ and $F^{\prime}$ are the measured positions of the principal and focal planes of the lens, $f^{\prime}$ is the experimental focal length, $\bar{f}^{\prime}$ is the mean value between both sides of each lens, $\delta f^{\prime}$ is $\bar{f}^{\prime}-f^{\prime}, \Delta f^{\prime}$ is the uncertainty of the linear fitting, and $\left|\Delta f^{\prime}\right| f_{n}^{\prime} \mid \%$ is the percentage experimental uncertainty.

\section{Conclusions}

In this work we present a simple and accurate technique to measure the focal length of a lens or lenses system. With this method, we are

Table 1

Nominal and experimental values for both measured lenses.

\begin{tabular}{|c|c|c|c|c|c|c|c|c|}
\hline Lens-side & $f_{n}^{\prime}(\mathrm{mm})$ & $H^{\prime}(\mathrm{mm})$ & $F^{\prime}(\mathrm{mm})$ & $f^{\prime}(\mathrm{mm})$ & $\bar{f}^{\prime}(\mathrm{mm})$ & $\delta f^{\prime}(\mathrm{mm})$ & $\Delta f^{\prime}(\mathrm{mm})$ & $\left|\Delta f^{\prime}\right| f_{n}^{\prime} \mid(\%)$ \\
\hline L1-front & +100.00 & -38.97 & 60.41 & +99.38 & +99.45 & -0.09 & \pm 0.092 & 0.092 \\
\hline L1-back & +100.00 & -1.70 & 97.85 & +99.55 & & +0.09 & \pm 0.072 & 0.072 \\
\hline L2-front & -100.00 & -27.65 & -127.81 & -100.16 & -100.23 & -0.07 & \pm 0.062 & 0.062 \\
\hline L2-back & -100.00 & -17.98 & -118.27 & -100.29 & & +0.07 & \pm 0.064 & 0.064 \\
\hline
\end{tabular}


able to determine the principal and focal planes of the lens and therefore the focal length. It is based on measuring the magnified/ demagnified period of the fringes produced by a diffraction grating at the near field after passing through the unknown lens. In addition, we use the variogram function to accurately calculate the period of the fringes, eliminating experimental noise and smoothing the self-images. We have checked two commercial lenses by Newport and we have experimentally obtained an accuracy around $0.1 \%$, one order of magnitude lesser than the uncertainty given by the manufacturer.

\section{Acknowledgments}

The authors thank J.A. Gomez-Pedrero for his help in this work. This work has been supported by project SPIP2015-01812 of the Ministerio de Interior of Spain and by SEGVAUTO- TRIES CM S2013/ MIT-2713 of the Dirección General de Universidades e Investigación, Comunidad de Madrid (Spain).

\section{References}

[1] Picht J. Mess-und prüfmethoden der optischen fertigung, 1. Berlin, Germany: Akademie-Verlag; 1955.

[2] Flügge J. Einführung in die Messung der optischen Grundgrößen. Karlsruhe, Germany: G. Braun; 1954.

[3] Dörband B, Gross H, Müller H. Handbook of optical systems. Metrology of optical components and systems, 5. Weinheim, Germany: John Wiley \& Sons; 2012.

[4] Malacara D. Optical shop testing. Wiley series in pure and applied optics. Hoboken, United States: Wiley; 2007.

[5] Nakano Y, Murata K. Talbot interferometry for measuring the focal length of a lens. Appl Opt 1985;24:3162-6.

[6] Jin X, Zhang J, Bai J, Hou C, Hou X. Calibration method for high-accuracy measurement of long focal length with Talbot interferometry. Appl Opt 2012;51:
2407-13.

[7] de Angelis M, de Nicola S, Ferraro P, Finizio A, Pierattini G. Analysis of moire fringes for measuring the focal length of lenses. Opt Lasers Eng 1998;30:279-86.

[8] Keren KM, Kreske E, Kafri O. Universal method for determining the focal length of optical systems by moire deflectometry. Appl Opt 1998;27(8):1383-9.

[9] Trivedi S, Dhanotia J, Prakash S. Measurement of focal length using phase shifted moiré deflectometry. Opt Lasers Eng 2013;51(6):776-82.

[10] Rasouli S, Rajabi Y, Sarabi H. Microlenses focal length measurement using z-scan and parallel moiré deflectometry. Opt Lasers Eng 2013;51(12):1321-6.

[11] Lee S. Talbot interferometry for measuring the focal length of a lens without moire fringes. J Opt Soc Korea 2015;19:165-8.

[12] Miks A, Pokorny P. Use of diffraction grating for measuring the focal length and distortion of optical systems. Appl Opt 2015;54(34):10200-6.

[13] Wu J-j, Chen J-b, Xu A-c, Gao X-y, Zhuang S. Focal length measurement based on Hartmann-Shack principle. Opt - Int J Light Electron Opt 2012;123(6):485-8.

[14] Tebaldi M, Forte G, Torroba R, Bolognini N, Tagliaferri A. Self-imaging pitch variation applied to focal length digital measurements. Opt Commun 2005;250(1): $10-5$.

[15] Mikš A, Pokorny ` P. Edge spread function of Talbot phenomenon. Opt - Int J Light Electron Opt 2016;127:8065-9. http://dx.doi.org/10.1016/j.ijleo.2016.06.003.

[16] Talbot HF. Lxxvi. Facts relating to optical science. No. iv. Lond Edinb Philos Mag J Sci 1836;9(56):401-7.

[17] Patorski K. I. The self-imaging phenomenon and its applications. Prog Opt 1989;27:1-108.

[18] Torcal-Milla FJ, Sanchez-Brea LM, Salgado-Remacha FJ, Bernabeu E. Self-imaging with curved gratings. Opt Commun 2010;283(20):3869-73.

[19] Torcal-Milla FJ, Sanchez-Brea LM, Bernabeu E. Near field diffraction of cylindrical convex gratings. J Opt 2015;17(3):035601.

[20] Morrison FA. Obtaining uncertainty measures on slope and intercept of a least squares fit with excel linest. Michigan, USA: Department of Chemical Engineering, Michigan Technological University, Houghton; 25 September 2014.

[21] Sanchez-Brea LM, Torcal-Milla FJ, Herrera-Fernandez JM, Morlanes T, Bernabeu E. Self-imaging technique for beam collimation. Opt Lett 2014;39(19):5764-7.

[22] Christensen R. Linear models for multivariate, time series, and spatial data. Berlin: Springer-Verlag; 1985.

[23] Cressie N. Statistics for spatial data, revised ed., vol. 928. New York: Wiley; 1993.

[24] Sanchez-Brea LM, Torcal-Milla FJ, Bernabeu E. Variogram-based method for contrast measurement. Appl Opt 2007;46(22):5027-33. 